\title{
Massive increase in monocyte HLA-DR expression can be used to discriminate between septic shock and hemophagocytic lymphohistiocytosis-induced shock
}

\author{
Solenn Remy ${ }^{1}$, Morgane Gossez ${ }^{2,3}$, Alexandre Belot ${ }^{4,5}$, Jack Hayman², Aurelie Portefaix ${ }^{6}$, Fabienne Venet ${ }^{2,3}$, \\ Etienne Javouhey ${ }^{1}$ and Guillaume Monneret ${ }^{2,3,7^{*}}$ (D)
}

Keywords: Immune response, Septic shock, Biomarker, Hemophagocytic lymphohistiocytosis

Clinical presentations of hemophagocytic lymphohistiocytosis (HLH) and septic shock share many similarities, including multiple organ dysfunction and overall clinical and biological symptoms. However, these life-threatening conditions require specific and opposing treatments. Currently, no single biomarker is available to differentiate septic shock from HLH at patient admission [1, 2]. HLH is classified as a primary (genetically inherited) or a secondary, i.e., induced by various inflammatory conditions (viral infections, autoimmune processes, lymphoid malignancies, or drug allergies), immune disorder. We report here the case of a young woman with febrile shock which proved to be a HLH caused by drug-induced hypersensitivity syndrome (DIHS). As septic shock was initially suspected, the patient benefited from broad immunological screening during the first week of evolution [3]. Strikingly, this revealed massively increased expression of monocyte human leukocyte antigen-DR (mHLA-DR) at 137,021 $\mathrm{ABC}$ (antibody bound per cell), even though expected values in septic shock are usually drastically decreased [3]. In addition, a positive response to increasing doses of corticosteroids was observed over time (Fig. 1). More precisely, while the patient's mHLA-DR expression was measured at $137,021 \mathrm{ABC}$ at admission, it decreased to $38,961 \mathrm{ABC}$ after the introduction of corticosteroids (day

\footnotetext{
* Correspondence: guillaume.monneret@chu-lyon.fr

${ }^{2}$ Hospices Civils de Lyon, Immunology Laboratory, E. Herriot Hospital, 69003 Lyon, France

${ }^{3} E A$ 7426, Pathophysiology of injury-induced immunosuppression (University Claude Bernard Lyon 1, BioMérieux, Hospices Civils de Lyon), E. Herriot

Hospital, 69003 Lyon, France

Full list of author information is available at the end of the article
}

3). Following the reactivation of inflammatory processes (day 5), mHLA-DR rose again $(66,829$ ABC). Finally, mHLA-DR returned to a normal range after increasing corticosteroid doses (20,499 ABC, day 8). All clinical features are provided in Additional file 1.

In the present patient, the extremely increased inaugural mHLA-DR value (i.e., 137,021 ABC) helped to unequivocally exclude a diagnosis of septic shock. Indeed, in our experience (more than 600 septic shock patients monitored over several years), the vast majority of mHLA-DR values measured within the first 3 days after septic shock are reported to be $<30,000 \mathrm{ABC}$ and mostly found below 10,000 ABC (normal values ranged from 15,000 to $40,000 \mathrm{ABC}$ ). This agrees with pathophysiology since HLH is secondary to overproduction of interferon- $\gamma$ (IFN- $\gamma)$, a cytokine known to be a strong inducer of mHLA-DR expression, whereas sepsis induces downregulation of mHLA-DR expression.

In conclusion, mHLA-DR may discriminate septic shock from HLH at admission despite both situations with multiple organ dysfunction sharing very common clinical and biological features (e.g., sCD25, elevated ferritin levels) $[4,5]$. This result obviously needs further assessment in various types of HLH. Upon confirmation, as these two deadly conditions (i.e., septic shock and HLH) would require opposing treatments, mHLA-DR may be of crucial help for clinicians regarding patients' care and management.

(C) The Author(s). 2018 Open Access This article is distributed under the terms of the Creative Commons Attribution 4.0 International License (http://creativecommons.org/licenses/by/4.0/), which permits unrestricted use, distribution, and reproduction in any medium, provided you give appropriate credit to the original author(s) and the source, provide a link to the Creative Commons license, and indicate if changes were made. The Creative Commons Public Domain Dedication waiver (http://creativecommons.org/publicdomain/zero/1.0/) applies to the data made available in this article, unless otherwise stated. 


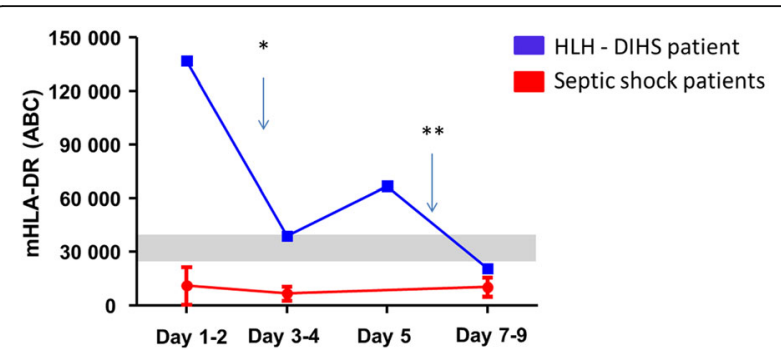

Fig. 1 Time course of $m H L A-D R$ in a HLH patient. Blue squares depict $m H L A-D R$ values in the HLH patient. Red circles represent pediatric septic shock values [3]. Gray range represents interquartile range values obtained previously in healthy children [3].

*Corticosteroids introduced, receiving $2 \mathrm{mg} / \mathrm{kg} /$ day; ${ }^{* *}$ corticosteroid adjustment to $4 \mathrm{mg} / \mathrm{kg} / \mathrm{day}$

\section{Additional file}

Additional file 1: Additional online information. (DOCX $27 \mathrm{~kb}$ )

\section{Abbreviations}

ABC: Antibody bound per cell; DIHS: Drug-induced hypersensitivity syndrome; HLH: Hemophagocytic lymphohistiocytosis; IFN- $\gamma$ : Interferon- $\gamma$; mHLA-DR: Monocyte human leukocyte antigen-DR

\section{Funding}

This work was supported by Hospices Civils de Lyon and University Claude Bernard Lyon 1.

\section{Availability of data and materials}

According to the French National Data Protection Commission, we are not authorized to provide the individual clinical data.

\section{Authors' contributions}

EJ and GM conceptualized and designed the PedIRIS study and reviewed and revised each draft of the manuscript. SR, AP, and AB participated in patient treatment and reviewed and revised the manuscript. SR, MG, FV, and $\mathrm{JH}$ performed biological analysis and reviewed and revised the manuscript. All authors read and approved the final manuscript.

\section{Ethics approval and consent to participate}

This case report belongs to the PedIRIS study. This observational prospective clinical study was approved by our Institutional Review Board (Comité de Protection des Personnes, Lyon Sud-Est II, number 2014-010-2, in accordance with Article L1121-1 of the French Public Health Code). According to legislation in place at the time of the study, this study required only the nonopposition of the study participants (written informed consent was not required). An information leaflet was systematically distributed to holders of parental authority. Participants and/or holders of parental authority could withdraw consent at any time. The study was registered: Pediatric Immune Response to Infectious Shock (PedIRIS), NCT02848144.

\section{Consent for publication}

Written informed consent for publication of their clinical details was obtained from the parent of the patient. A copy of the consent form is available for review by the Editor of this journal.

\section{Competing interests}

The authors declare that they have no competing interests.

\section{Publisher's Note}

Springer Nature remains neutral with regard to jurisdictional claims in published maps and institutional affiliations.

\section{Author details}

Hospices Civils de Lyon, Paediatric Intensive Care Unit, Mother and Children University Hospital, 59 Boulevard Pinel, 69500 Bron, France. ${ }^{2}$ Hospices Civils de Lyon, Immunology Laboratory, E. Herriot Hospital, 69003 Lyon, France.

${ }^{3}$ EA 7426, Pathophysiology of injury-induced immunosuppression (University Claude Bernard Lyon 1, BioMérieux, Hospices Civils de Lyon), E. Herriot Hospital, 69003 Lyon, France. ${ }^{4}$ Hospices Civils de Lyon, Paediatric Nephrology, Rheumatology, Dermatology Unit, National Referee Centre for pediatric-onset Rheumatism and autoimmune diseases (RAISE), Mother and Children University Hospital, 59 Boulevard Pinel, 69500 Bron, France.

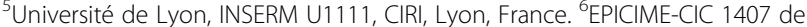
Lyon, Inserm, Service de Pharmacologie Clinique, CHU-Lyon, Bron, France. ${ }^{7}$ Cellular Immunology Laboratory, Hôpital E. Herriot - Hospices Civils de Lyon, France Pavillon E - 5 place d'Arsonval, 69437 Lyon, Cedex 03, France.

Received: 20 July 2018 Accepted: 30 July 2018

Published online: 11 September 2018

\section{References}

1. Castillo L, Carcillo J. Secondary hemophagocytic lymphohistiocytosis and severe sepsis/ systemic inflammatory response syndrome/multiorgan dysfunction syndrome/macrophage activation syndrome share common intermediate phenotypes on a spectrum of inflammation. Pediatr Crit Care Med. 2009;10(3):387-92.

2. Machowicz R, Janka G. Similar but not the same: differential diagnosis of HLH and sepsis. Crit Rev Oncol Hematol. 2017:114:1-12.

3. Remy S, Kolev-Descamps K. Occurrence of marked sepsis-induced immunosuppression in pediatric septic shock: a pilot study. Ann Intensive Care. 2018;8(1):36.

4. Rosario C, Zandman-Goddard G. The hyperferritinemic syndrome: macrophage activation syndrome, Still's disease, septic shock and catastrophic antiphospholipid syndrome. BMC Med. 2013;11:185.

5. Møller HJ, Moestrup S. Macrophage serum markers in pneumococcal bacteremia: prediction of survival by soluble CD163. Crit Care Med. 2006; 34(10):2561-6. 